\title{
Ruido magnético Barkhausen para el estudio de la anisotropia magnética en aceros inoxidables
}

\section{Magnetic Barkhausen noise for the study of magnetic anisotropy in stainless steels}

\footnotetext{
${ }^{1}$ Dpto. Proyecto ICES y Ondas Elásticas, Comisión Nacional de Energía CNEA, Av. G. Paz 1499, San Martín, Buenos Aires, Argentina.

${ }^{2}$ Universidad Tecnológica Nacional UTN, Fac. Reg. Delta, Buenos Aires, Argentina.

${ }^{3}$ IT Sabato, UNSAM-CNEA, Av. G. Paz 1499, San Martin, Buenos Aires, Argentina.

${ }^{4}$ Universidad Nacional de Tres de Febrero, UNTREF, Ingeniería de Sonido, Buenos Aires, Argentina.

${ }^{5}$ Universidad Nacional de Chilecito, UNdeC, Chilecito, La Rioja, Argentina.

${ }^{6}$ Departamento de Engenharia Mecânica, Escola Politécnica, Universidade de São Paulo, São Paulo, Brasil. e-mail: neyra@cnea.gov.ar,nnunez@cnea.gov.ar, lopezpum@cnea.gov.ar, ruzzante@gmail.com, 1rpadove@usp.br
}

\section{RESUMEN}

El ruido magnético Barkhausen (RMB) es un fenómeno que ocurre en materiales ferromagnéticos cuando se someten a campos magnéticos lentamente variables, generándose cambios en su magnetización, produciéndose "saltos" discretos. Estos saltos se generan por el movimiento de las paredes de los dominios magnéticos. Se detectan colocando una pequeña bobina sobre la superficie de la muestra. El movimiento de las paredes es regulado por la microestructura del material (precipitados, tamaño de grano, tensiones residuales, etc.). Es esta manera indirecta la que permite caracterizar al material. En este trabajo se presentan las mediciones del RMB para estudiar la anisotropía magnética, inducida por el proceso de laminación, y los cambios producidos por deformación plástica uniaxial en probetas de aceros inoxidables ferríticos AISI: 409, 430, 441A. Las mediciones se realizaron a diferentes porcentajes de deformación, con el fin de observar cómo cambian las características de las señales de RMB. Se presentan también los estudios métalográficos y de dureza. Los resultados muestran una clara tendencia en relación con la dirección de los esfuerzos principales.

Palabras clave: Ruido magnético Barkhausen, acero inoxidable, anisotropía magnética, deformación plástica.

\section{ABSTRACT}

The magnetic Barkhausen noise (MBN) is a phenomenon that occurs in ferromagnetic materials when they are exposed to slowly varying magnetic fields, generating discrete jumps in their magnetization. These jumps are produced by the movement of the magnetic domain walls. They can be detected by a small coil allocated on the sample surface. The movement of the domain wall is regulated by the microstructure of the material (precipitates, grain size, residual stresses, etc.). Through this indirect way it is possible to characterize the material. In this work MBN measurements to study magnetic anisotropy induced by rolling processes and uniaxial strain tests are shown for ferritic stainless steels AISI: 409, 430 and 441A. The measurements were made at different strain percentages. The objective was to follow the changes in the MBN signals. The metallographic and hardness studies are presented. The results show a clear trend related with the direction of the principal stresses.

Keywords: Magnetic Barkhausen noise, stainless steel, magnetic anisotropy, plastic deformation.

\section{INTRODUCCIÓN}

El ruido magnético Barkhausen (RMB) es una importante técnica no destructiva para ensayo de materiales ferromagnéticos. Se han hecho muchos estudios relacionándolo con la microestructura de los aceros, lo que permite investigar el estado de los mismos, incluyendo su orientación cristalográfica. La anisotropía magnética es la no homogeneidad de las propiedades magnéticas al ser medidas en diferentes direcciones del mate- 
rial. Si la población de granos exhibe una orientación preferencial, conocida como textura, el material también puede exhibir mayor anisotropía magnética. Las propiedades magnéticas de un material dependen de la dirección en la que son medidas. Sólo la anisotropía magnetocristalina que está directamente relacionada con la simetría del cristal es una propiedad intrínseca del material, mientras que las otras anisotropías magnéticas (de forma, de intercambio, de tensiones, etc.) son extrínsecas o "inducidas". El diseño de los materiales ferromagnéticos comerciales depende de su anisotropía magnética [1-7]. Por estas razones el RMB es una técnica adecuada para su detección, aún desconociendo el proceso de fabricación. Como resultado, cuando se mide el valor RMS (valor cuadrático medio) de las señales de RMB, en función de la dirección del campo magnético externo aplicado, se puede estudiar la anisotropía magnética del material. La forma de la gráfica correspondiente revela la dirección del eje de fácil magnetización, ya que la señal RMB es mayor en esa dirección en particular. En ausencia de la anisotropía magnética, la gráfica del valor RMS es una circunferencia. $\mathrm{Si}$, en cambio, sobre el material se aplica una carga de tracción uniaxial, la circunferencia se alarga en esa dirección, manifestando su anisotropía magnética que coincide con la dirección de la carga de tracción, transformando la circunferencia en una elipse [2]. Sin embargo, en el caso del esfuerzo de compresión se observa la disminución del valor angular del RMB en la dirección de la compresión, sin embargo el eje de fácil magnetización sigue estando presente y ese efecto es aún más pronunciado en el rango plástico de deformación $[2,3]$.

Las diferentes etapas del proceso de deformación plástica pueden ser estudiadas usando el RMB. Las dislocaciones producidas por deformación plástica generan: a) una distribución no homogénea de campos de tensiones a nivel microscópico, contribuyendo a la redistribución de las tensiones en los granos y también de las tensiones intergranulares; b) cambios en la textura cristalográfica que puede alterar la anisotropía magnética del material. La combinación de estos cambios introduce variaciones en las señales RMB porque ellos determinan la dinámica del movimiento de las paredes de los dominios [5].

En este trabajo se presentan las mediciones de RMB en placas de aceros inoxidables ferríticos, para estudiar la anisotropía magnética inducida por el proceso de laminación y por deformación en ensayos de tracción uniaxial con diferentes porcentajes de deformación. También se muestran las micrografías y los estudios de dureza e histéresis magnética.

\section{MATERIALES Y MÉTODOS}

En esta sección se describen los materiales utilizados, su caracterización y el sistema de medición del RMB.

\subsection{Materiales}

Los materiales ensayados son aceros inoxidables ferríticos AISI: 409, 430 y 441A, fabricados por el Grupo Arcelor Mittal Inox de Brasil y entregados en forma de placas (300 mm x $210 \mathrm{~mm}$ x 0,5 mm), laminadas en frio y recocidas. Para cada material se prepararon 5 probetas para ensayos de tracción uniaxial, bajo la Norma ASTM E8, cortadas en la dirección perpendicular al sentido de laminación. Una de ellas fue ensayada hasta rotura para estudiar las propiedades mecánicas de cada material [8]. El resto fue traccionado hasta distintos porcentajes de deformación final. En la Figura 1 se muestra una fotografía de la probeta indicando sus dimensiones.

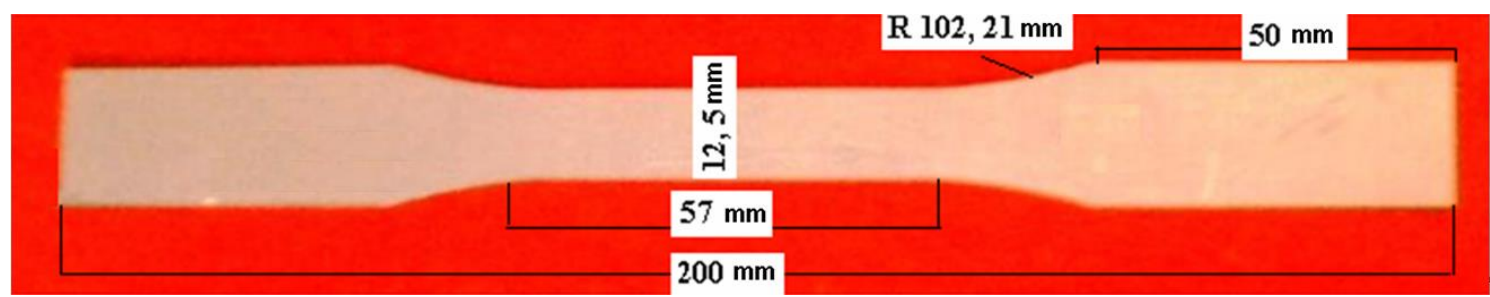

Figura 1: Fotografía de una probeta con sus dimensiones.

\subsection{Deformación plástica}

Para los ensayos de tracción se utilizó una máquina universal de ensayos (EMIC) controlada por PC con el programa Tesc versión 3.04 (ver Figura 2). Las 4 probetas de un mismo material fueron deformadas hasta una deformación de $\mathrm{D}_{1}$; una de ellas fue separada y las tres restantes fueron sometidas hasta una deformación $\mathrm{D}_{2}$; nuevamente una fue separada y las otras fueron ensayadas hasta $\mathrm{D}_{3}$; finalmente, una sola fue llevada has- 
ta la deformación $\mathrm{D}_{4}$. Durante la deformación se midió la carga instantánea aplicada (con una celda de carga de $50 \mathrm{kN}$ ) y el alargamiento resultante con un extensómetro Trd 6, de $50 \mathrm{~mm}$ de apertura inicial. Los ensayos se realizaron a una velocidad constante de $1 \mathrm{~mm} / \mathrm{min}$, siguiendo la norma ASTM E-8. En cada probeta previamente apartada se midió la anisotropía magnética en la zona de mayor deformación (centro de la probeta) y la microdureza Vickers con una carga de $0,2 \mathrm{~kg}$. Luego se cortó de la misma zona un pequeño trozo para el estudio de la microestructura con microscopia óptica y otro para realizar la determinación del ciclo de histéresis. Todas las probetas fueron atacadas electrolíticamente con ácido oxálico al $10 \%$ con una tensión de 10 $\mathrm{V}$ durante un $1 \mathrm{~min}$.

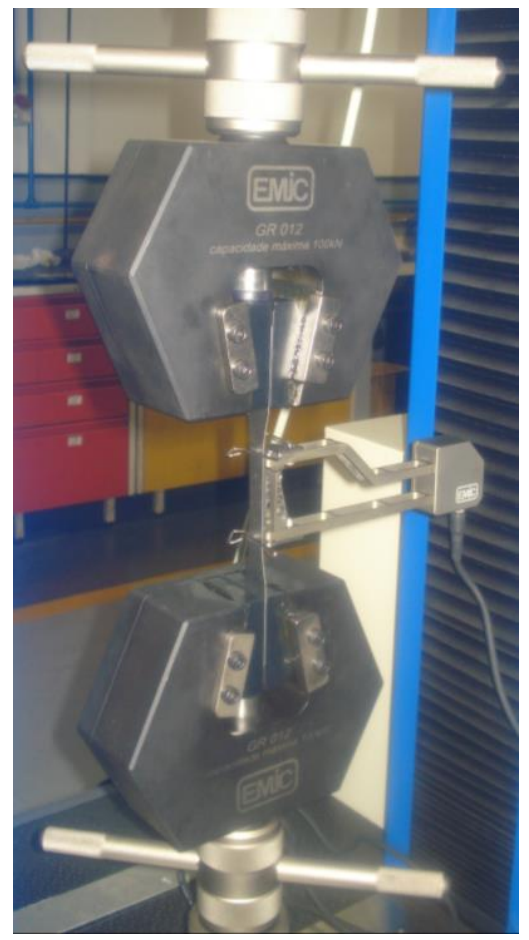

Figura 2: Fotografía de una probeta en la máquina de ensayos.

\subsection{Medición del RMB}

Una vez alcanzada cada una de las deformaciones $\mathrm{D}_{1}$ a $\mathrm{D}_{4}$ se desmontaron las probetas y las mediciones de RMB se realizaron en el laboratorio del Departamento ICES y Ondas Elásticas.

El campo magnético de excitación se logró con una corriente sinusoidal de $10 \mathrm{~Hz}$ producida por un generador de funciones LeCroy ARB Studio 1102, amplificada con un amplificador de potencia. Esta corriente se utilizó para excitar la bobina del yugo. La bobina sensora del RMB se colocó en el centro del yugo. El amplificador de esta bobina sensora tiene una banda de paso de $5 \mathrm{kHz}$ a $200 \mathrm{kHz}$. Tanto el yugo como la bobina sensora fueron encerrados en una cajita de aluminio (ver Figuras 3y 4). Para mejorar la relación señal-ruido cada probeta fue conectada a tierra.

La probeta se ubicó sobre la dirección $90^{\circ}-270^{\circ}$ del gráfico polar, de modo que la dirección de laminación coincidiera con la dirección $0^{\circ}-180^{\circ}$. Al mismo tiempo la dirección de $0^{\circ}$ coincide con el campo magnético de excitación inicial. Luego se varió la dirección del campo girando el yugo cada $30^{\circ}$ hasta completar la circunferencia.

Sobre la placa original, antes del corte de las probetas, se registró el RMB cada $15^{\circ}$, para identificar la anisotropía producida por el proceso de fabricación. La medición angular del RMB en las placas originales se realizó con un yugo de mayor tamaño, pero se debió construir uno más pequeño para las probetas de tracción debido a la geometría de las mismas. En la Figura 3 se muestra una fotografía de todo el sistema de medición del RMB y en la Figura 4, una vista amplificada de la medición angular del RMB de una probeta. Todas las señales fueron grabadas en un osciloscopio LeCroy digital, en el canal 1 la señal RMB y en el canal 2 el voltaje de excitación sobre una resistencia de 10 Ohm. 


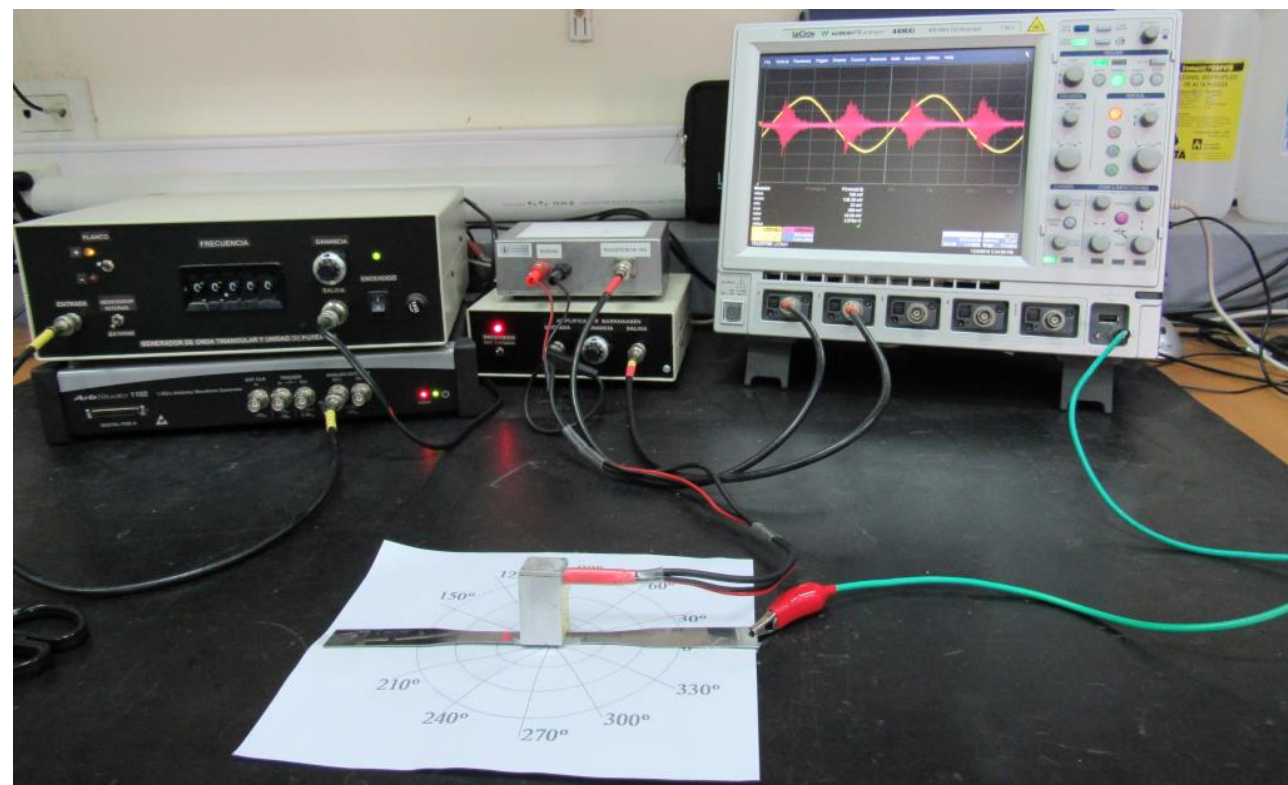

Figura 3: Esquema del sistema experimental de medición del RMB.

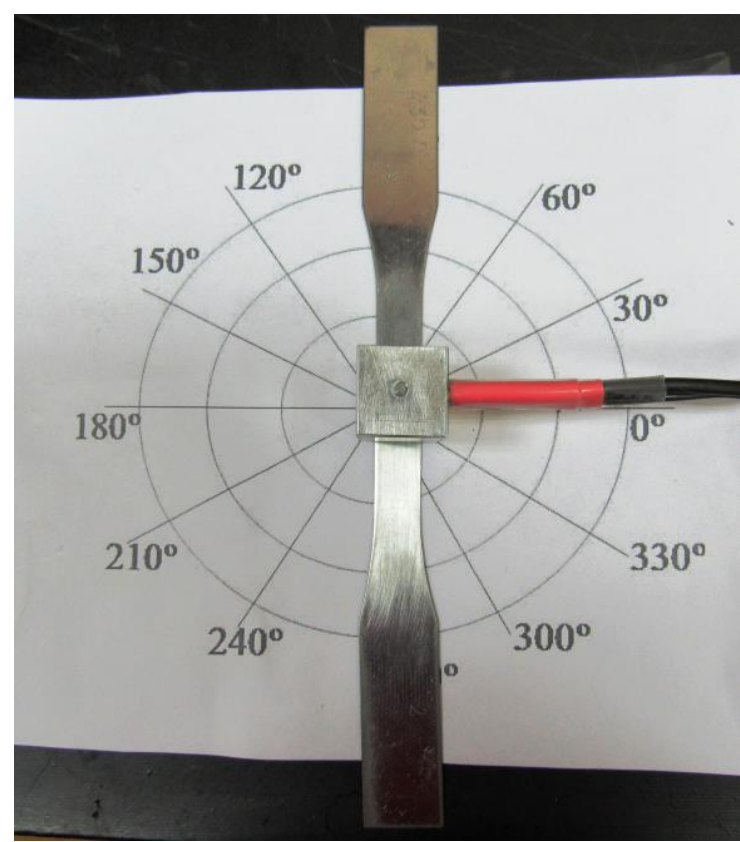

Figura 4: Fotografía de una vista amplificada del sistema de medición del RMB en una probeta.

\section{RESULTADOS Y DISCUSIÓN}

En las siguientes dos secciones se detallan los resultados del análisis microestructural y el de las mediciones magnéticas.

\subsection{Análisis microestructural}

En la Figura 5 se muestran los resultados obtenidos por microscopia óptica del material sin deformar, para los tres aceros. Estas placas fueron laminadas en frio y recocidas con un espesor final de 0,5 mm. Las mismas presentan una microestructura constituida por una matriz ferrítica y granos recristalizados en forma equiaxial, libres de deformación. Para los tres materiales, no se manifestó nítidamente la deformación producida por la laminación ya que no se ven granos alargados. El acero 409 (a) presenta pequeños granos recristalizados que varían de $10 \mu \mathrm{m}$ a $100 \mu \mathrm{m}$; el 430 (b) muestra un tamaño de grano uniforme de $10 \mu \mathrm{m}$ a $20 \mu \mathrm{m}$ y el $441 \mathrm{~A}$ (c) exhibe un tamaño de grano dúplex con variación entre de $5 \mu \mathrm{m}$ a $100 \mu \mathrm{m}$ [9]. 

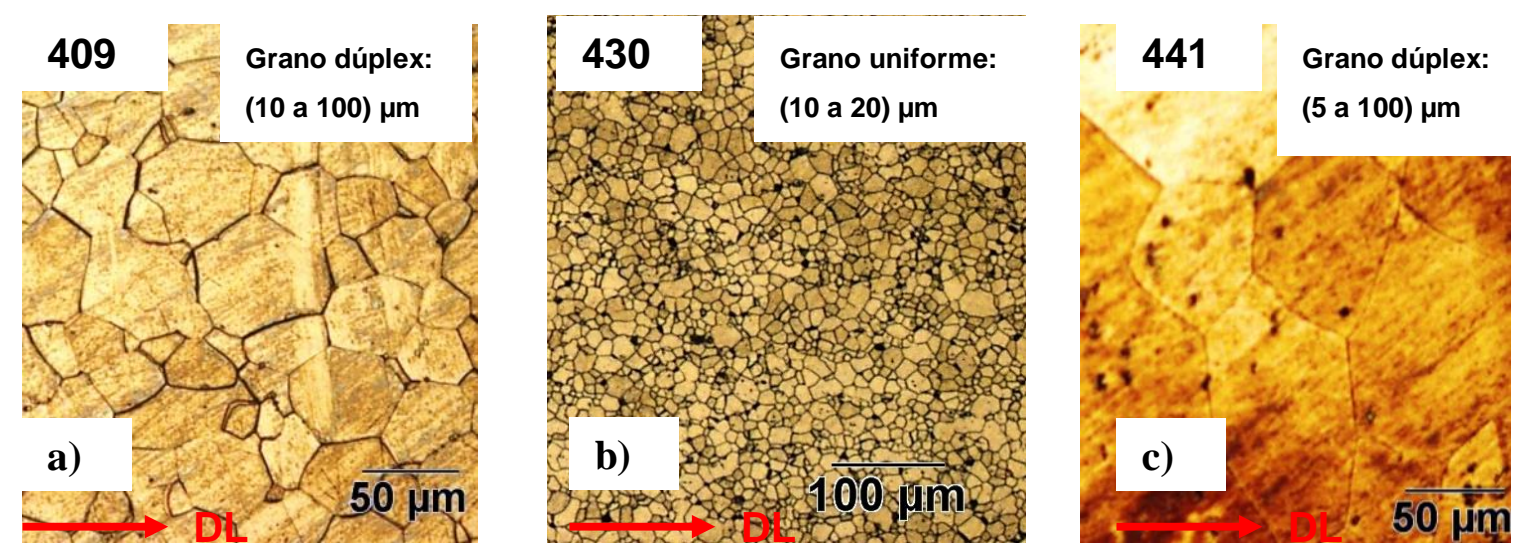

Figura 5: Fotografías de las microscopias ópticas de las probetas de los aceros inoxidables AISI a) 409, b) 430 y c) 441 A sin deformar. La flecha roja indica el sentido de laminación.

En la Figura 6 se muestran las metalografías del acero AISI 409, para las probetas deformadas en los ensayos de tracción uniaxial: a) $5 \%$, b) $10 \%$, c) $15 \%$ y d) $20 \%$. Se observa que a medida que aumenta la deformación los granos crecen relativamente pero no pierden su forma equiaxial.
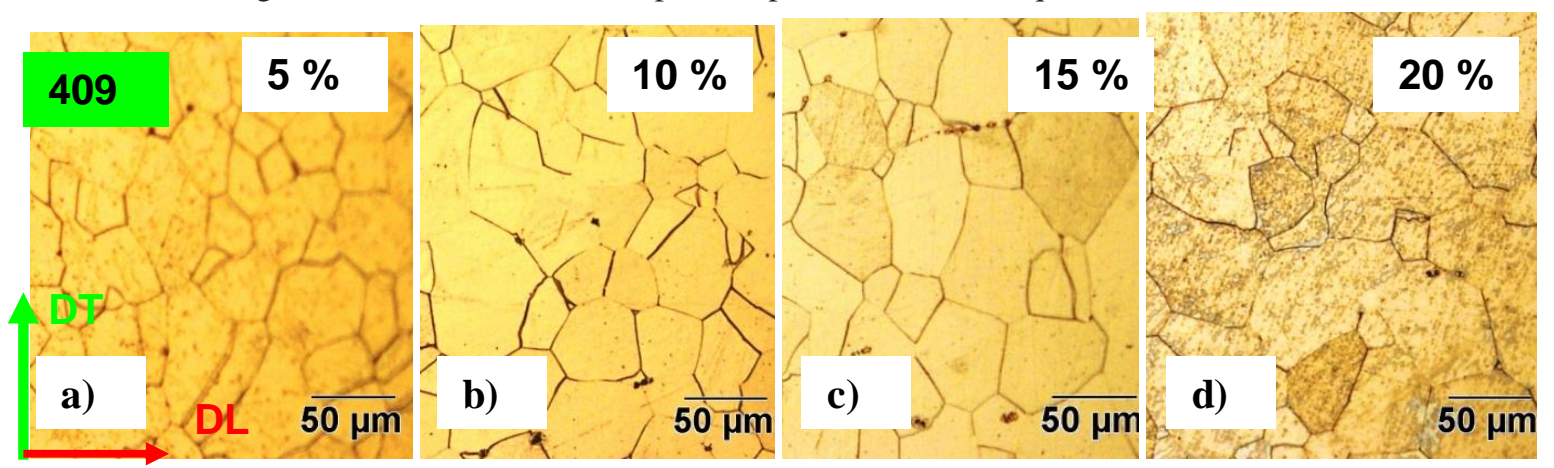

Figura 6: Fotografías de las microscopías ópticas del acero inoxidable AISI 409, para diferentes porcentajes de deformación a) $5 \%$, b) $10 \%$, c) $15 \%$ y d) $20 \%$. La flecha roja indica el sentido de laminación (DL) y la fecha verde el sentido de la tracción (DT).

En la Figura 7 se muestran las metalografías del acero AISI 430, para las probetas deformadas en los ensayos de tracción uniaxial: a) $5 \%$, b) $8 \%$, c) $9 \%$ y d) $13 \%$. No se observan cambios significativos a medida que aumenta la deformación.
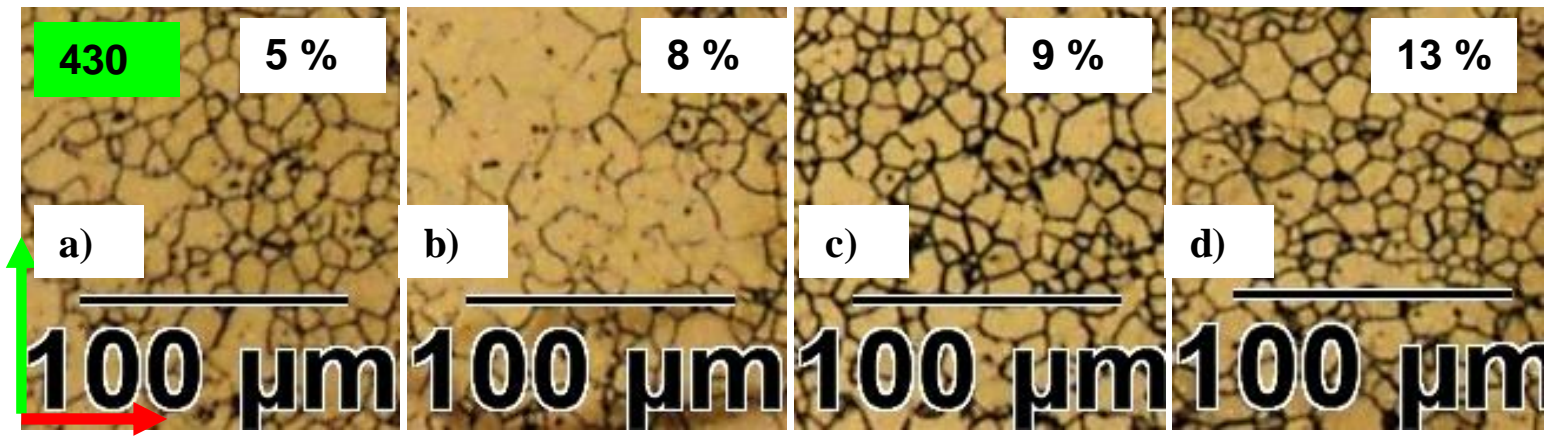

Figura 7: Fotografías de las microscopías ópticas del acero inoxidable AISI 430, para diferentes porcentajes de deformación: a) $5 \%$, b) $8 \%$, c) $9 \%$ y d) $13 \%$. La flecha roja indica el sentido de laminación (DL) y la verde el sentido de la tracción (DT).

En la Figura 8 se muestran las metalografías del acero AISI 441A, para las probetas deformadas: a) $3 \%$, b) $4 \%$, c) $5 \%$ y d) $7 \%$. Debido a que las deformaciones no son muy grandes no se distinguió un nítido alargamiento del grano en la dirección de tracción (DT). 

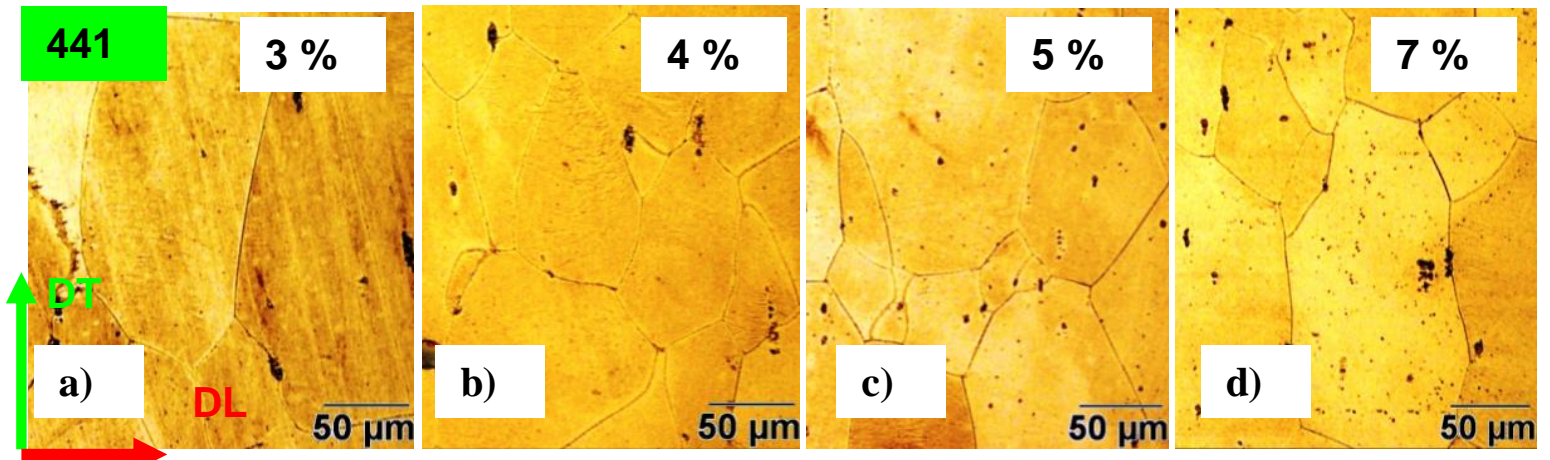

Figura 8: Fotografías de las microscopias ópticas del acero inoxidable AISI 441A, para diferentes porcentajes de deformación: a) $3 \%$, b) $4 \%$, c) $5 \%$ y d) $7 \%$. La flecha roja indica el sentido de laminación (DL) y la verde el sentido de la tracción (DT).

En la Tabla 1 se muestran los resultados de la microdureza Vickers para cada probeta con los diferentes porcentajes de deformación. Se observa que hay un aumento de la microdureza con el aumento de la deformación plástica, debido al endurecimiento del material. El mecanismo exacto del endurecimiento no se conoce con precisión, pero durante la deformación se crean nuevas dislocaciones que interactúan con las ya existentes, creando enredos de dislocaciones. La densidad de dislocaciones aumenta con la deformación, haciendo que sea más difícil el movimiento de éstas a través de las dislocaciones ya existentes y entonces el material disminuye su ductilidad y se endurece [10].

Tabla 1: Mediciones de microdureza Vickers para cada probeta en los diferentes porcentajes de deformación.

\begin{tabular}{|c|c|c|c|c|c|}
\hline \multicolumn{2}{|c|}{ AISI 409 } & \multicolumn{2}{c|}{ AISI 430 } & \multicolumn{2}{c|}{ AISI 441A } \\
\hline $\begin{array}{c}\text { Deformación } \\
(\%)\end{array}$ & $\begin{array}{c}\text { Microdureza } \\
\text { Vickers } \\
\left(\mathrm{HV}_{0,2}\right)\end{array}$ & $\begin{array}{c}\text { Deformación } \\
(\%)\end{array}$ & $\begin{array}{c}\text { Microdureza } \\
\text { Vickers } \\
\left(\mathrm{HV}_{0,2}\right)\end{array}$ & $\begin{array}{c}\text { Deformación } \\
(\%)\end{array}$ & $\begin{array}{c}\text { Microdureza } \\
\text { Vickers } \\
\left(\mathrm{HV}_{0,2}\right)\end{array}$ \\
\hline 0 & 164 & 0 & 198 & 0 & 226 \\
\hline 5 & 195 & 5 & 207 & 3 & 237 \\
\hline 10 & 211 & 8 & 209 & 4 & 254 \\
\hline 15 & 222 & 9 & 219 & 5 & 256 \\
\hline 20 & 230 & 13 & 229 & 7 & 259 \\
\hline
\end{tabular}

\subsection{Estudios magnéticos}

En la Figura 9 se muestra el diagrama polar del valor RMS del RMB para cada acero inoxidable. Estos valores fueron medidos sobre las placas originales, tal como fueron entregadas por el fabricante, antes de los ensayos de deformación. El propósito de estas mediciones fue corroborar la dirección de laminación (DL) previamente indicada por el fabricante. En a) para el 409 la forma de la gráfica revela la existencia de anisotropía magnética, la señal de RMB es mayor en DL, coincidiendo con la dirección del eje de fácil magnetización y corroborándose así la indicación del fabricante. En b) para el 430 el eje de fácil magnetización medido (mayores valores de RMB) resultó perpendicular a la DL indicada por el fabricante, revelándose una discrepancia, que involucra una rotación de $90^{\circ}$. En c) para el $441 \mathrm{~A}$, se observa una distribución casi uniforme, no presentando una anisotropía magnética claramente distinguible, debido a que es el material con mayor dureza (Tabla 1). 

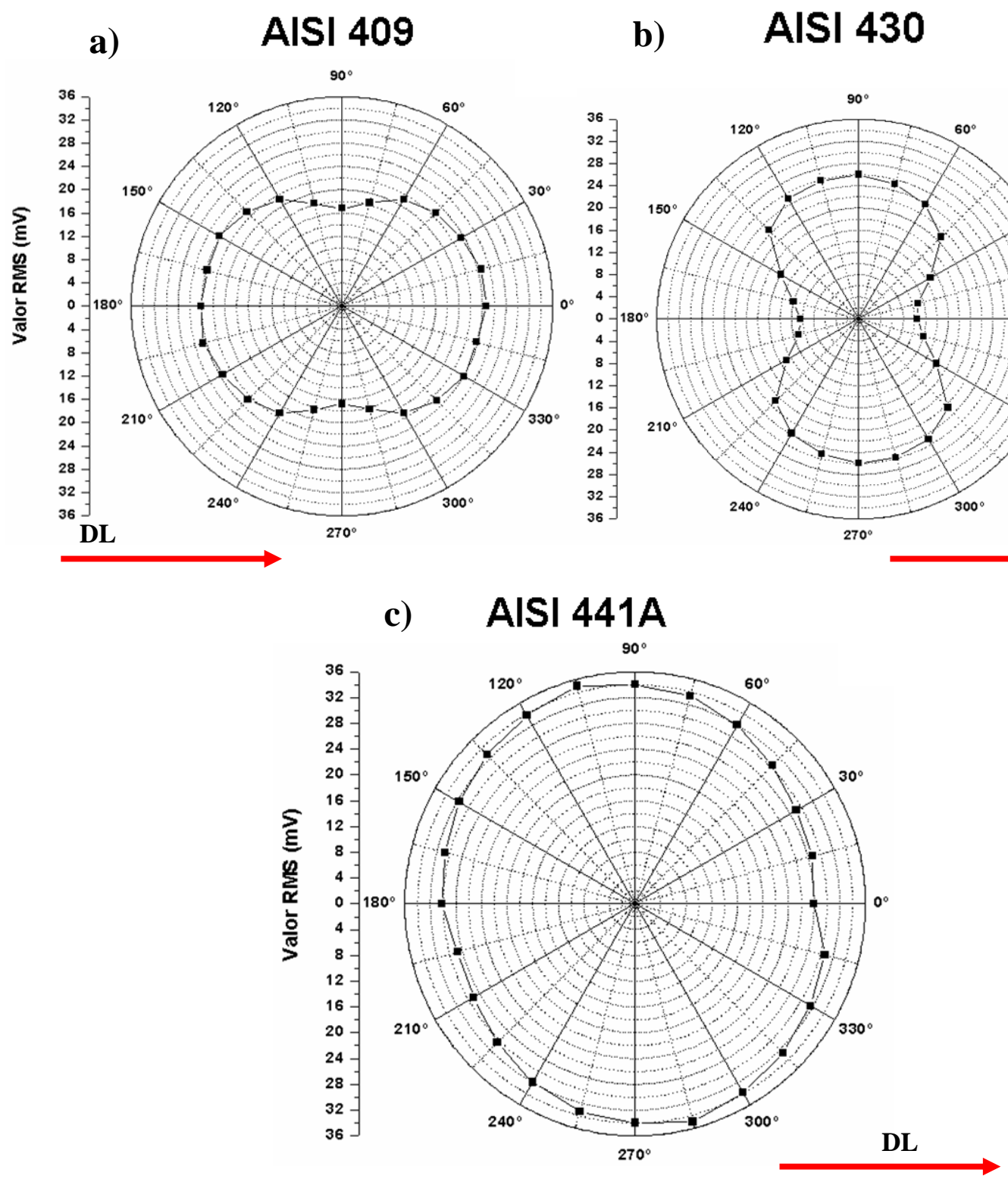

Figura 9: Mapa de anisotropía magnética usando el valor RMS de las señales de RMB en las placas de aceros inoxidables AISI a) 409, b) 430 y c) 441A. La flecha negra indica la dirección de laminación (DL) tal como lo indica el fabricante.

De las placas rectangulares se cortaron en la dirección perpendicular a la DL, 4 probetas para su posterior ensayo de tracción uniaxial. Como ya se dijo, las 4 probetas de un mismo material fueron deformadas hasta una deformación $\mathrm{D}_{1}$; una de ellas fue separada y las tres restantes fueron sometidas hasta una deformación $\mathrm{D}_{2}$; se procedió del mismo modo hasta quedar con una sola probeta con deformación $\mathrm{D}_{4}$.

En la Figura 10, se muestra el diagrama polar del valor RMS de las señales de RMB, para los aceros con las cuatro deformaciones, medido sobre cada probeta separada previamente. Se observa que la anisotropía magnética inducida en este proceso coincide con la dirección de tracción (DT), aumentando constantemente el RMB con la deformación, incrementándose también la dureza (ver Tabla 1). 
AISI 409

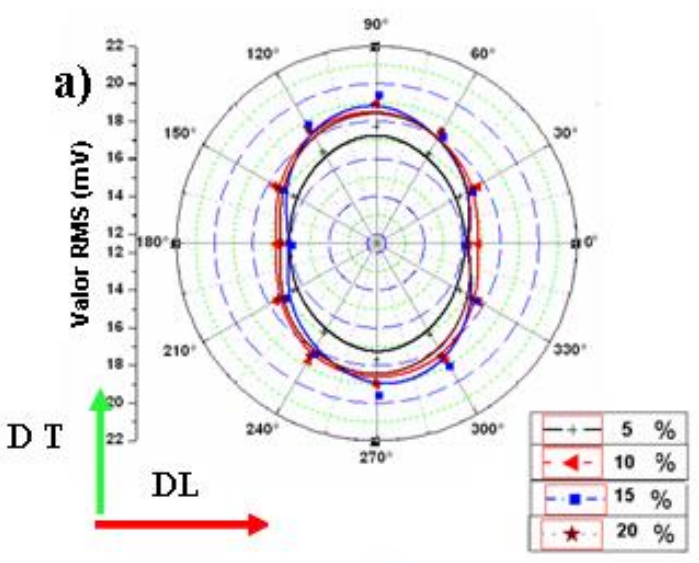

AISI 430

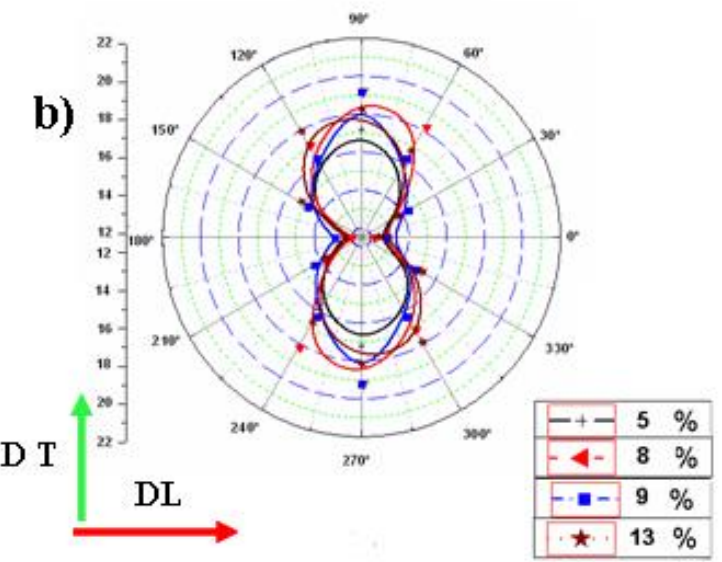

\section{AISI 441A}

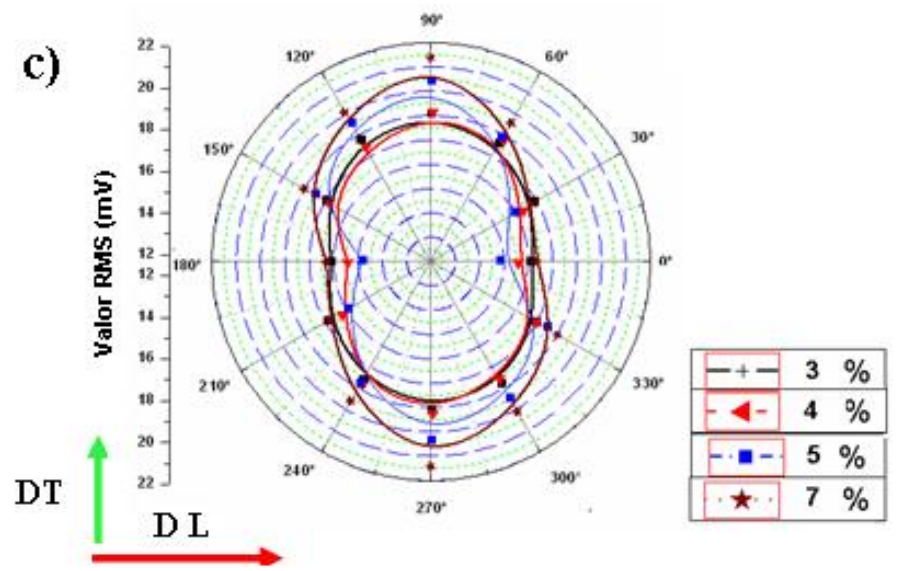

Figura 10: Mapa de anisotropía magnética usando el valor RMS en las probetas traccionadas de los aceros inoxidables AISI a) 409, b) 430 y c) 441A, para los diferentes porcentajes de deformación axial.

En las Figuras 10 (a) y (c), para el AISI 409 y 441A se observa, comparando con las Figuras 9 (a) y (c), que al aumentar la deformación plástica se produce un eje de fácil magnetización coincidente con la dirección de tracción (DT), pero perpendicular a la DL, es decir hay una rotación. Esto puede ser explicado por el hecho de que en el proceso de laminación se provocan tensiones residuales compresivas en dirección longitudinal y de tracción en la dirección perpendicular a la dirección de deformación. Debido al fenómeno de la magnetostricción inversa, los dominios magnéticos tienden a alinearse paralelamente a las tensiones de tracción y perpendicularmente a tensiones compresivas (ver Figura 11), lo que resulta en una mayor cantidad de dominios magnéticos alineados perpendicularmente a dirección de laminación al final del proceso [1, 2, 11].
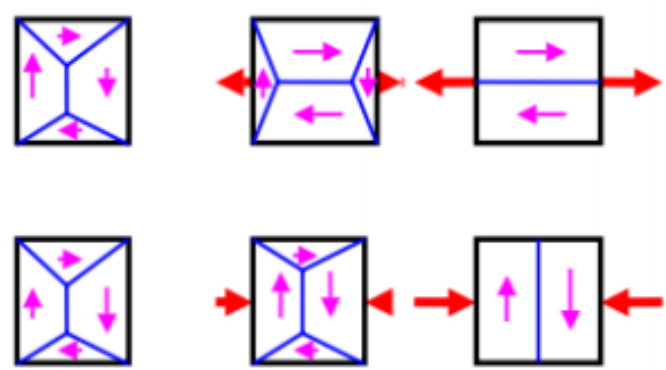

Figura 11: Efecto magnetoelástico: los dominios se alinean paralelamente a la dirección de tracción y perpendicularmente a la dirección de compresión [11]. 
En el caso del 430 (b), ahora sí se observa la anisotropía con mayores valores de RMB en la dirección de tracción (DT). Esto último induciría a pensar, que en este caso particular, la dirección DL aportada por el fabricante en realidad estaría rotada $90^{\circ}$.

De acuerdo a la curva tensión deformación, las deformaciones realizadas en estos aceros se encuentran en la primera región posterior a la fluencia [12]. En esta región, la estructura de las dislocaciones cambia significativamente y homogéneamente en una vista macroscópica. Los resultados de RMB están claramente afectados por estos cambios en la densidad de dislocación, pero sus detalles son menos claros. Parece existir una relación sutil entre pequeños defectos como dislocaciones y el RMB. En general, se supone que un mayor número de defectos aumenta la actividad del RMB, ya que habrá más repentinos movimientos de las paredes de los dominios al saltar esas barreras de potencial [13]. Sin embargo, si estos defectos actúan como puntos de anclajes fuertes, evitan que las paredes del dominio se muevan en absoluto y así disminuirá la actividad del RMB.

Por otra parte, si los defectos son demasiado débiles, no actuarán como puntos de anclaje, pero amortiguarán el movimiento, por lo tanto hacen más lentos los cambios repentinos, lo que resulta en una menor actividad del RMB. Para las dislocaciones relativamente aisladas, todavía no está claro si los defectos suficientemente grandes puedan actuar como puntos de anclaje y, por lo tanto si aumentará o disminuirá la actividad del RMB. La resistencia de fijación de dislocaciones aisladas y enredos de dislocaciones son posibles. Las dislocaciones aisladas podrían tener suficiente fuerza para actuar como puntos de anclajes, lo que significa que su presencia aumentará el RMB. Alternativamente, las dislocaciones aisladas podrían tener fuerza de fijación insuficiente para actuar como puntos de anclajes, pero actúan como amortiguadores de la velocidad de movimiento de las paredes de los dominios, lo que significa que su presencia disminuiría el RMB [1, 2 , 13].

Para profundizar el análisis del comportamiento magnético de estos materiales se realizaron mediciones de sus ciclos de histéresis para el material original y después de cada deformación. Estas se llevaron a cabo con el Magnetómetro de Muestra Vibrante, Modelo Versalab, Marca Quantum Design, del Departamento de Física de la Materia Condensada, Gerencia de Investigación y Aplicaciones, GAIyANN de la Comisión Nacional de Energía Atómica (CNEA). En la Figura 12 se muestra a modo de ejemplo, la superposición del ciclo de histéresis del acero AISI 441A, para una muestra sin deformar y otra deformada al $7 \%$. Se ve que a medida que aumenta el porcentaje de deformación plástica, se produce una rotación del ciclo de histéresis en el sentido anti horario. Esto ocurre con el aumento de la densidad de dislocaciones, y la consecuente formación de puntos de anclaje, que dificultan el movimiento de las paredes de los dominios magnéticos resultando un aumento en la saturación magnética, la remanencia y la coercitividad. Cuanto mayor es la coercitividad, más fuerte es la obstrucción al movimiento de las paredes de los dominios durante el proceso de magnetización [11-14]. En todos los casos se verificó el mismo comportamiento. Es importante destacar que si bien la Figura 12 da idea de que la fuerza coercitiva es cero para la magnetización nula, al hacer una ampliación de la curva en esa zona, se observa que es distinta de cero. Si bien no se presenta aquí el gráfico del ciclo de histéresis para el 430, la información aportada por el técnico, indicaba también un comportamiento rotado $90^{\circ}$ respecto de la DL aportada por el fabricante. 


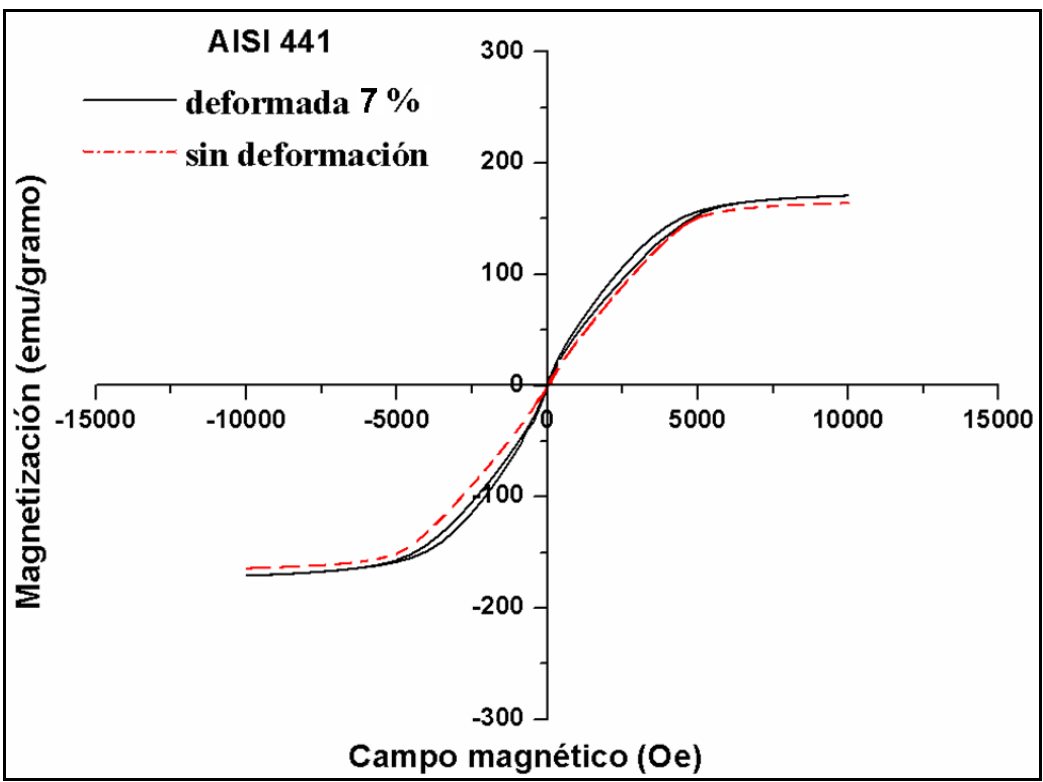

Figura 12: Curvas de histéresis: AISI 441A con $0 \%$ y con $7 \%$ de deformación.

\section{CONCLUSIONES}

Se midió y se estudió la anisotropía magnética en placas de tres aceros inoxidables ferríticos AISI: 409, 430 y 441A, mediante mediciones de RMB en las placas originales y en probetas de ensayos de tracción uniaxiales sometidas a distintas deformaciones.

Se presentaron las imágenes de microscopias óptica y los valores de los ensayos de dureza Vickers para cada material, en cada etapa de deformación, relacionándolos entre sí.

Se comprobó mediante los diagramas polares correspondientes a cada deformación, la aparición de la dirección de fácil magnetización paralela al eje de tracción.

En el caso del acero 430, se encontró una discrepancia respecto a la dirección de laminación indicada por el fabricante, presumiéndose una rotación de $90^{\circ}$. Este último hecho fue corroborado también con el ciclo de histéresis del material virgen.

En todos los ensayos de tracción se vio que al aumentar la deformación aumentaba la anisotropía, obteniéndose el eje mayor del gráfico polar coincidente con la dirección de deformación.

En etapas posteriores de esta investigación está previsto realizar análisis de textura mediante difracción de rayos X, profundizándose en la relación entre las señales de RMB y las características microestructurales de cada material.

\section{AGRADECIMIENTOS}

A Joaquín Sacanell por los estudios de histéresis magnética, Departamento de Física de la Materia Condensada, Gerencia de Investigación y Aplicaciones, GAIyANN - CAC - CNEA, Argentina. A la División Metalografía y Ensayos Termomecánicos, Gerencia Materiales, Comisión Nacional de Energía Atómica, Buenos Aires, Argentina.

\section{BIBLIOGRAFÍA}

[1] CULliTY B. D, GRAHAM C. D., Introduction to Magnetic Materials, 2da Edition, Published by John Wiley \& Sons, Inc., Hoboken, New Jersey, United States, 2009.

[2] STEFANITA C. G., From Bulk to Nano. The Many Sides of Magnetism, Springer Series in Materials Science, 2008.

[3] MARU, M, PADOVESE, L., PEREZ-BENITEZ, J., et al., "Evaluation Plastic Deformation by the Magnetic Barkhausen Noise", ECNDT, pp. 1-14, 2006.

[4] KRAUSE, T., CAPHAM, L., PATTANTYUS, A, et al., "Investigation of the stress-dependent magnetic easy axis in steel using magnetic Barkhausen noise”, Journal Applied Physics, v. 79, pp. 4242- 4252, 1996.

[5] CAMPOS, M F., CAMPOS, M A., LANDGRAF, F J G., et al., "Anisotropy study of grain oriented steels 
with Magnetic Barkhausen Noise”, Journal of Physics: Conference Series, v.303, pp. 1-6, 2011.

[6] AMIRI, M. S., RABUNG, M., THIELEN,M., et al., "On the role of crystal and stress anisotropy in magnetic Barkhausen noise", Journal of Magnetism and Magnetic Materials, v.372, pp.16-22, November 2014.

[7] DHAR, A., CLAPHAM, L., ATHERTON, D. L, "Influence of uniaxial plastic deformation on magnetic Barkhausen noise in steel”, NDT-E International, v. 34, pp. 507-514, 2001.

[8] AStUdillo, M. R. N., NÚÑEZ, N., PUMAREGA, M.I.L., et al., "Ruido Magnético Barkhausen y Emisión Magneto Acústica en Ensayos de Tensión Uniaxiales en Placas de Acero Inoxidable", In: Congreso Internacional de Metalurgia y Materiales $14^{\circ}$ SAN CONAMET/ XIII IBEROMAT /Materia 2014, $1^{\mathrm{a}}$ ed. Santa Fe: Universidad Nacional del Litoral, 2014, Santa Fe, Argentina, 21-24 de octubre, 2014.

[9] AStUdillo, M. R. N,, NÚÑEZ, N., PUMAREGA, M.I.L., et al., "Magnetic Barkhausen Noise and Magneto Acoustic Emission in Stainless Steel Plates", Procedía Materials Science, v.8, pp. 674-682, 2015.

[10] CALLISTER, W., "Introducción a la ciencia de los materiales”, Editorial Reverte, S. A, Barcelona, 2002.

[11] PRAXEDES, T. O., "Sistema Experimental para Realização de Ensaios Não Destrutivos Magnéticos e Sua Aplicação na Avaliação da Degradação Microestrutural Do Aço A516 Grau 60", Dissertação de M.Sc., Universidade Federal do Ceará, Fortaleza, 2012.

[12] ASTUDILlO, M. R. N., SÁNCHEZ, G.L, NÚÑEZ, N., et al., "Caracterización no Destructiva de Aceros inoxidables usando Ruido Magnético Barkhausen y Emisión Magneto Acústica”, In: 20 Conferencia del Acero IAS 2014, 5-6 de noviembre del 2014, Rosario, Argentina.

[13] VOLKERS, R., "The influence of dislocations on the magnetic properties in steel”, Masters Thesis, Delf University of Technology, Paises Bajos, 2008.

[14] ÜMIT AKÇAOĞLU, F., HAKAN GÜR, C. "Monitoring Magnetic Anisotropy Variations in ColdRolled Steels by Magnetic Barkhausen Noise Method", In:11th European Conference on Non-Destructive Testing, Prague, Czech Republic, October 6-10, 2014. 